\title{
Enhancement and Registration Schemes for Matching Conjunctival Vasculature ${ }^{\star}$
}

\author{
Simona Crihalmeanu ${ }^{1}$, Arun Ross ${ }^{1}$, and Reza Derakhshani ${ }^{2}$ \\ 1 West Virginia University, Morgantown WV 26506, USA \\ ${ }^{2}$ University of Missouri, Kansas City, MO 64110, USA
}

\begin{abstract}
Ocular biometrics has made significant strides over the past decade primarily due to the rapid advances in iris recognition. Recent literature has investigated the possibility of using conjunctival vasculature as an added ocular biometric. These patterns, observed on the sclera of the human eye, are especially significant when the iris is off-angle with respect to the acquisition device resulting in the exposure of the scleral surface. In this work, we design enhancement and registration methods to process and match conjunctival vasculature obtained under non-ideal conditions. The goal is to determine if conjunctival vasculature is a viable biometric in an operational environment. Initial results are promising and suggest the need for designing advanced image processing and registration schemes for furthering the utility of this novel biometric. However, we postulate that in an operational environment, conjunctival vasculature has to be used with the iris in a bimodal configuration.
\end{abstract}

\section{Introduction}

The eyes have played a significant role in biometrics primarily due to the unique and stable features that can be extracted from the the iris, a circular diaphragm forming the colored portion of the eye. It is well established that the iris texture is a reliable biometric, with numerous forms of variability across individuals and is believed to be stable over time when imaged in the near-IR spectrum 3 . However, when the iris becomes "off-angle" with respect to the focal plane of the camera, the sclera (also known as the white of the eye) is revealed. Sclera, the external layer of the eye, is a firm dense membrane comprising of a white and opaque fibrin connective tissue, organized in many bands of parallel and interlacing fibrous tissue bundles. Its outer surface, called episclera, contains the blood vessels nourishing the sclera. The anterior part of the sclera is covered by the conjuctival membrane - a thin membrane containing secretory epithelium that helps lubricate the eyes for eyelid closure. The focus of this work is on the rich vasculature pattern revealed in the episclera and conjunctiva membrane hereafter referred to as the conjunctival vasculature. In a previous study [2], images of 12 eyes pertaining to the left and right eyes of 6 subjects were used to study the possibility of using this pattern as a biometric. In this study, we

\footnotetext{
* This work was funded by the Center for Identification Technology Research (CITeR).
} 
conduct a more elaborate experiment involving 50 subjects in order to determine the suitability of using the conjunctival vasculature as a biometric. The primary contribution is the design of image enhancement and registration schemes to match conjunctival vasculature obtained in a non-ideal environment containing ambient lighting.

The paper is divided as follow: section 2 describes data acquisition; section 3 discusses sclera segmentation; section 4 describes specular reflection detection and removal; section 5 describes methods to enhance the image; section 6 discusses the registration method, and section 7 describes the experimental results.

The block diagram of the proposed system is shown in Fig 1

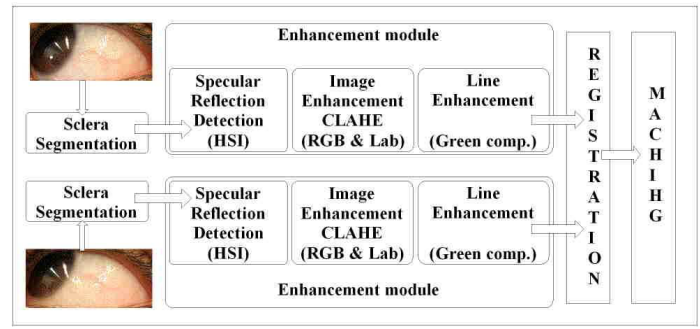

Fig. 1. Block diagram for enhancement and registration of conjunctival vasculature

\section{Image Acquisition}

The SONY CyberShot DSC F717(5 megapixels) was used to capture color images of the eye. Each subject was asked to move their eyes in the following manner with respect to the optical axis of the camera: frontal, upward, to the left, and to the right. Thus, different regions of the sclera were represented in the ensuing pictures. These color images (RGB) were collected in two sessions. The first session had 2400 images from 50 subjects, and the second session had 816 images from 17 of the original 50 subjects. Images were captured from both eyes at three different distances: 1 feet considered as near distance images, 9 feet as medium distance images and 12 feet as far distance images. For each eye, 2 images were collected per gaze at each distance (Fig. 2).

The paper presents the results on a subset of images belonging to the "left-eyelooking-left near-distance" category that contains 100 images from 50 subjects in the first session, and 34 images from 17 of the 50 subjects in the second session.

\section{Sclera Region Segmentation}

Image segmentation refers to the procedure that partitions the image into regions of interest according to pixel intensity values, based on either discontinuities (abrupt changes in intensity values such as edges) or similarities (such as grouping pixels based on predefined criteria). Accurately segmenting the sclera from 


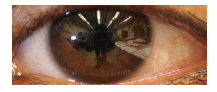

(a)

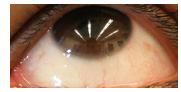

(b)

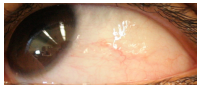

( c)

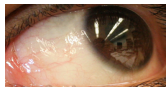

(d)

Fig. 2. Near images of the eye where the subject is: a) looking straight ahead, b) looking up, c) looking left, and d) looking right with respect to the camera

the eye image is very important for further processing. A semi-automated technique is used for this purpose. The proposed technique first applies an automated clustering method whose output is subsequently refined by manual intervention. Each pixel is represented as a three-dimensional point in a Cartesian coordinate system based on its primary spectral components of red, green and blue. The $k$-means clustering algorithm is used to partition the pixels into three categories. Since the sclera is typically whiter than the rest of the eye, such a procedure is expected to work well in separating the scleral pixels from the rest of the image. The output of clustering is a binary mask, which when imposed on the original image, will identify the region of interest corresponding to the sclera.

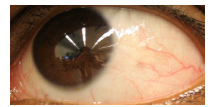

(a)

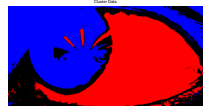

(b)

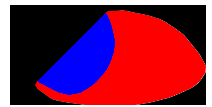

(c)

Fig. 3. Segmenting the sclera from two different eye images, displayed by column: a) Original image, b) Segmented sclera region based on RGB values (red = sclera region, blue $=$ iris region, black $=$ the background) $\mathrm{c}$ ) Convex hull of the sclera (blue+red) containing a portion of the iris (blue)

The sclera pixel cluster is determined as that cluster which has the largest Euclidean distance from the origin of the coordinate system to its centroid. The iris pixel cluster is determined as that cluster with the smallest Euclidean distance from the origin of the coordinate system to its centroid. Entries marked as ' 1 ' in the mask denote the pixels assigned to the sclera cluster. Due to the image characteristics of illumination and specular reflection, it is possible for some pixels from the sclera to not be assigned to the proper cluster, thereby appearing as holes in the mask. In order to eliminate these holes, the convex hull of the pixels in the sclera cluster is considered Fig. 3 (c). This, however, means that pixels pertaining to the iris cluster may be included in the mask. To address this, we first remove those pixels within the convex hull belonging to the iris cluster. Next, we remove the convex hull of the iris pixels included in the mask. Fig. 目(c) shows the segmented color image, after imposing the mask on the image.

On examining the segmented sclera region, we observed that in some images, a small portion of the lower eyelid was erroneously included. To address this issue,

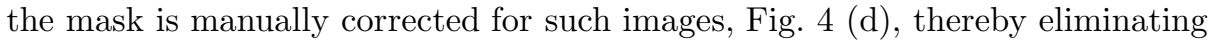
the lower eyelashes, Fig. 目 (e). 


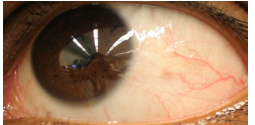

(a)

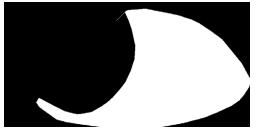

(b)

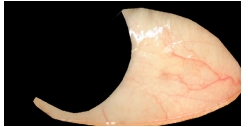

(c)

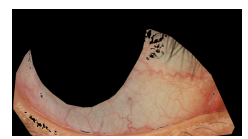

(d)

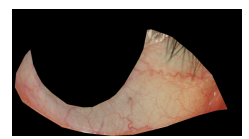

(e)

Fig. 4. 1) Sclera region segmentation: a) Original image, b) Sclera mask, c) Segmented sclera region. 2) Manual correction of segmented sclera: d) Original image, e) Segmented sclera region after manual correction.

Table 1. Manual correction statistics of segmented sclera

\begin{tabular}{|c|l|c|c|c|c|}
\hline Gaze & Distance & \multicolumn{2}{|c|}{ Left eyes } & \multicolumn{2}{c|}{ Right eyes } \\
\cline { 3 - 6 } & $\begin{array}{c}\text { automatic } \\
\text { segmentation } \\
\text { and manual } \\
\text { correction }\end{array}$ & $\begin{array}{c}\text { only automatic } \\
\text { segmentation }\end{array}$ & $\begin{array}{c}\text { automatic } \\
\text { segmentation } \\
\text { and manual } \\
\text { correction }\end{array}$ & $\begin{array}{c}\text { only automatic } \\
\text { segmentation }\end{array}$ \\
\hline \multirow{3}{*}{ Looking left } & near & 61 & 73 & 42 & 92 \\
\cline { 2 - 6 } & medium & 49 & 85 & 36 & 98 \\
\cline { 2 - 6 } & far & 43 & 91 & 39 & 95 \\
\hline \multirow{3}{*}{ Looking right } & near & 54 & 80 & 56 & 78 \\
\cline { 2 - 6 } & medium & 53 & 81 & 51 & 93 \\
\cline { 2 - 6 } & far & 35 & 99 & 44 & 90 \\
\hline
\end{tabular}

Table 1 records the number of images for which manual correction of the segmented sclera was needed.

\section{Specular Reflection}

\subsection{General Considerations}

Specular reflections may provide valuable information about the shape of the object and its location with respect to the light source. However, they can cause problems for image processing algorithms that may erroneously consider these specularities as pixels of interest during the process of segmentation resulting in spurious results. Localization of specularities in images is very important and requires a good understanding of the reflection of light, a complicated process that depends on the material of the object under consideration, the roughness of its surface, the angle of illumination, the angle of viewing, and the wavelength of light. 
Specular reflections on the sclera have different topologies, sizes and shapes that cannot be described by a single pattern. Their pixel intensity values are distinctively high, and exhibit a large variation both within the same image and across multiple images. Different approaches for specular reflection detection and removal have been proposed in the literature $[8,4,, 9],[1],[10,[6], 1]$. The algorithm for specular reflection, consists of three main steps: detection and localization of specular reflection, construction of specular reflection mask, and exclusion of the region containing the specular reflection from the sclera region.

\subsection{Detection of Specular Reflection}

If original images of sclera containing specular reflection were to be further processed, as explained in the following sections, the edges of the specular reflection region may appear as spurious blood vessels in the enhanced image. Our algorithm to detect specular reflections is based on the power law transformation as applied to pixel intensities in the color image. Power law transformations have the basic form:

$$
S=c * R^{\gamma}
$$

where $\mathrm{c}, \gamma$ are positive constants, $\mathrm{R}$ is the input pixel intensity, and $\mathrm{S}$ is the output intensity. As shown in Fig. 5, by simply varying $\gamma$ we obtain a family of possible transformation curves. For $\gamma>1$, the power - law curves map a narrow range of light input values into a wider range of output values. For $\gamma<1$, the power - law curves map a narrow range of dark input values into a wider range of output values. In order to detect specularities, we consider $\gamma$ an integer in the range $[1,10]$.

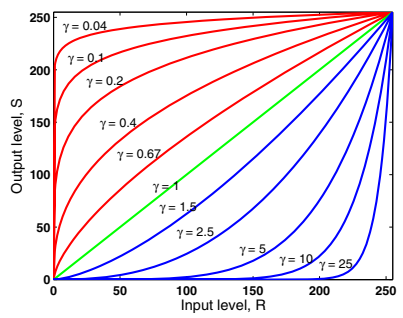

Fig. 5. Plots of the power-law transform for various values of $\gamma ; \mathrm{c}=1$ in all cases

The algorithm is described by the following steps:

1) Convert the RGB image to the HSI (hue, saturation, illumination) color space.

2) Consider the illumination component of the HSI space as the input image R in equation 1 .

3) Compute the output image $\mathrm{S}$ for different $\gamma$ values using equation 1 . Fig. 6 (a) displays results for $\gamma=3$. 
4) Compute the histogram for each image $S$ as seen in Fig. 6 (b).

5) Compute the filtered histogram for each image $S$ using the moving average [1/3 $1 / 3$ 1/3] filter as seen in Fig. 6] (c).

6) Compute the slope $\theta$ of the filtered histogram.

7) For the filtered histogram corresponding to each $\gamma$, find the first negative $\theta$ $\left(\theta_{\gamma}\right)$ and its corresponding intensity value, $I_{\gamma}$, as a potential threshold value for detecting specular reflection.

8) Examine the distribution of $\theta_{\gamma}$ as a function of $\gamma$ to select $\gamma_{o p t}$, $\gamma_{o p t}$ $=\arg \max \left(\left|\theta_{\gamma}-\theta_{\gamma-1}\right|\right)$. Fig. 7 shows $\gamma_{o p t}=5$; for near distance images, the threshold to detect specular reflection is selected as the mean of all thresholds values found for $\gamma, 5 \leq \gamma \leq 10$.

9) Use the threshold value found to obtain a binary mask for isolating specular reflection.

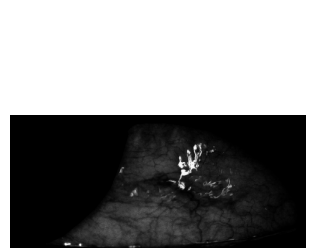

(a)

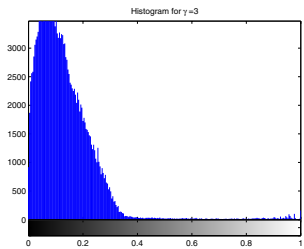

(b)

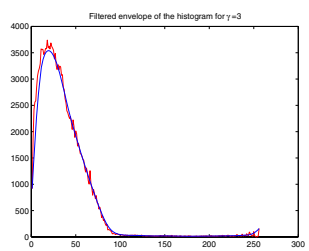

(c)

Fig. 6. Detection of specularities. Examples for $\gamma=3$ : (a) Illumination component of HSI sclera image; (b) Histogram of the illumination component; (c) Filtered envelop of the histogram.

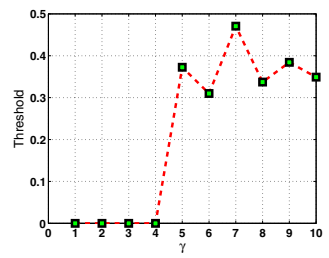

Fig. 7. Threshold values as a function of $\gamma$ for an image.

Fig 8 shows the results of specular reflection detection.

\subsection{Segmented Sclera Image without Specular Reflection}

The segmented sclera image without specular reflection is obtained as follows:

1) Use sclera mask and specular reflection mask to obtain the final sclera mask without specular reflection.

2) Superimpose the final mask on the RGB image to obtain segmented sclera without specular reflection (Fig 9). 


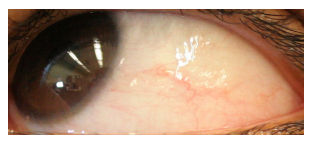

(a)

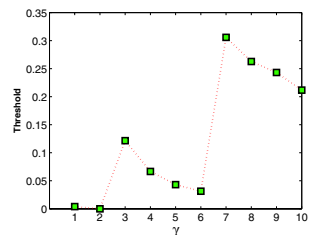

(b)

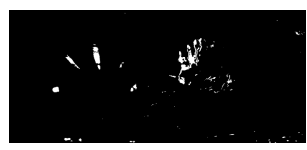

(c)

Fig. 8. Detecting specularities: a) Original image, b) Threshold values for $1 \leq \gamma \leq 10$ c) Specular reflection mask

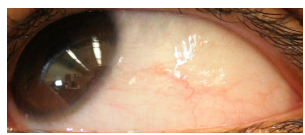

(a)

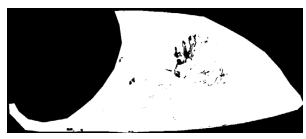

(b)

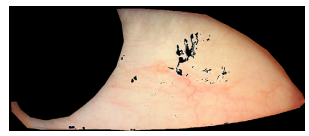

(c)

Fig. 9. Segmenting the sclera after removing specularities: a) Original image, b) Specular reflection mask c) Segmented sclera without specular reflection

\section{Image Pre-processing}

Images of the conjunctival vasculature present a large variation in intensity values and offer a low contrast between vessels and the background. Therefore sclera veins are pre-processed in two consecutive steps. The first one involves color image enhancement using contrast-limited adaptive histogram equalization (CLAHE) that operates and enhances the contrast on small regions of the color image called tiles and then combines neighboring tiles using bilinear interpolation to eliminate artificially induced boundaries (Fig. 10 (b)).

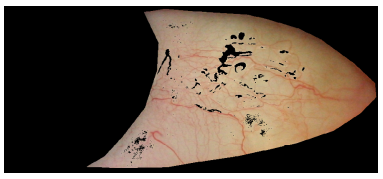

(a)

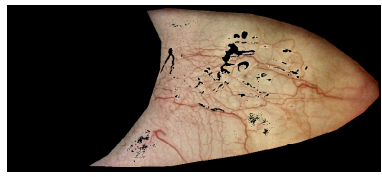

(b)

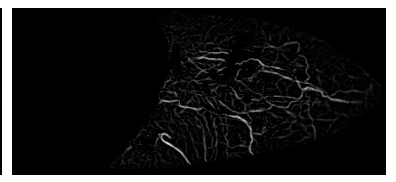

(c)

Fig. 10. Image enhancement: (a) Original sclera vein image, (b) Enhanced sclera vein image; Line enhancement: (c) Example of pre-processed sclera vein image

In order to obtain the best results for vein segmentation, an examination of the three components (red, blue and green) of the enhanced colored sclera images, suggests the use of the green component that has the best contrast between the blood vessels and the background. In order to improve sensitivity to vein detection and segmentation, we use a selective enhancement filter for lines, as described in [5]. The result is a normalized image with enhanced blood vessels as seen in Fig. 10 (c). 


\section{Sclera Image Registration}

Image registration is the process of finding a transformation that aligns one image to another. The method used here, described in 7], models a local affine and a global smooth transformation. It also accounts for contrast and brightness variations between the two images that are to be registered. The registration between two images, the source $f(x, y, t)$ and the target $f(\hat{x}, \hat{y}, t-1)$, is modeled by the transformation $\boldsymbol{m}=\left(m_{1}, m_{2}, m_{3}, m_{4}, m_{5}, m_{6}, m_{7}, m_{8}\right)$ :

$$
m_{7} f(x, y, t)+m_{8}=f\left(m_{1} x+m_{2} y+m_{5}, m_{3} x+m_{4} y+m_{6}, t-1\right) .
$$

where $m_{1}, m_{2}, m_{3}$, and $m_{4}$ are the linear affine parameters, $m_{5}, m_{6}$ are the translation parameters, and $m_{7}, m_{8}$ are the contrast and brightness parameters. A multi-scale approach is employed by using a Gaussian pyramid to downsample the images to be registered. From the coarse-to-fine level, the transformation $\boldsymbol{m}$ is determined globally as well as locally at each level of the pyramid and the estimated parameters are used to warp the source image. Fig. [1] shows results for the registration of two pre-processed sclera images.

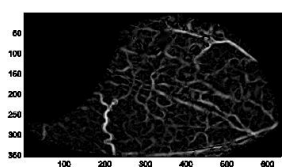

(a)

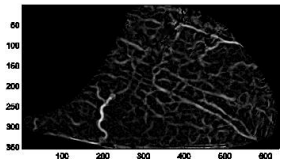

(b)

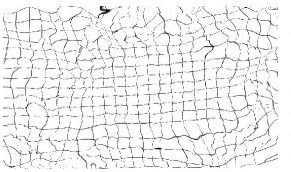

(c)

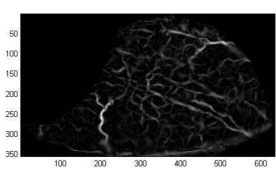

(d)

Fig. 11. Registering images: (a) Target image, (b) Source image, (c) Rectified warp, and (d) Registered source image

Using the linear affine parameters $m_{1}, m_{2}, m_{3}$, and $m_{4}$, and the translation parameters $m_{5}, m_{6}$, the sclera mask of the source image is also registered. The result is a grayscale image that is transformed into a binary image via a threshold, $T h=(M+m) / 2$ where $M$ is the maximum value of the registered mask and $m$ is its minimum value.

\section{Matching Results}

The similarity between two sclera images is assessed using cross-correlation between regions of the sclera that do not include the specular reflections from both images. Results are displayed in Fig 12 .

This initial analysis indicates that an EER of $\sim 25 \%$ has been obtained on the database of 50 subjects. An assessment of the results suggests that the large intraclass variation is caused by the following factors: (a) the large range of viewing angles; (b) harsh ambient lighting resulting in significant specularities; and (c) the curved ocular surface that cannot be modeled using a linear surface. In our previous paper 2 we manually cropped portions of the sclera and performed matching. In this paper, the entire segmented region of the sclera was considered. Further, the issue of specular reflection and image enhancement were addressed. 


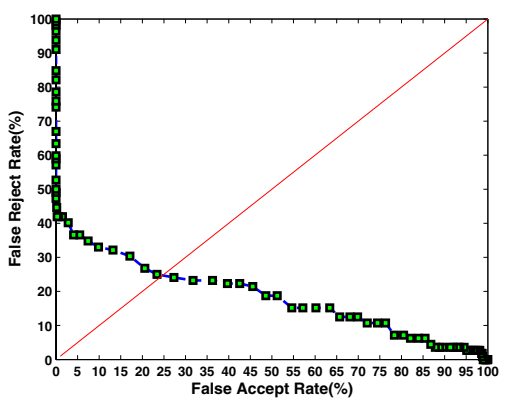

Fig. 12. ROC curve indicating the matching performance for the left-eye looking-left images obtained at a near distance

\section{Summary and Future Work}

The use of conjunctival vasculature in the biometric field is a fairly new idea. In this work, we designed image enhancement and registration schemes for processing the conjunctival vasculature information pertaining to 50 subjects. The images used in this work were taken without any constraints on lighting thereby exhibiting a wide range of irregularities including blurring and specularities. An EER of $25 \%$ was obtained on the eye images labeled as left-eye looking-left obtained at the near distance. We are currently conducting experiments on the remaining eye images. There are several challenges associated with processing these images. These issues are related to: (a) the curved surface of the eye, (b) the glossy surface of the sclera that reflects light, (c) the presence of less prominent veins that can degrade performance, and (d) eyelashes that obstruct the sclera region and can be incorrectly perceived as vasculature patterns. We are currently developing methods to handle these challenging images. We are also looking at ways to combine the sclera with the iris for enhanced biometric recognition. We conclude this paper by noting that more work is essential to make conjunctival vasculature a practical biometric that can operate in non-ideal environments.

\section{References}

1. Brelstaff, G., Blake, A.: Detecting Specular Reflections Using Lambertian Constraints. In: Second International Conference on Computer Vision, pp. 297-302 (1988)

2. Derakhshani, R., Ross, A., Crihalmeanu, S.: A New Biometric Modality Based On Conjunctival Vasculature. Artificial Neural Networks in Engineering, St. Louis, USA (2006)

3. Daugman, J.: How Iris Recognition Works. IEEE Transactions on Circuits and Systems for Video Technology 14(1), 21-30 (2004)

4. Klinker, G.J., Shafer, S.A., Kanade, T.: Using a Color Reflection Model to Separate Highlights from Object Color. In: First International Conference on Computer Vision, pp. 145-150 (1987) 
5. Li, Q., Sone, S., Doi, K.: Selective enhancement filters for nodules, vessels, and airway walls in two-and three dimentional CT scans. Medical Physics 30(8) (2003)

6. Park, J.B.: Detection of Specular Highlights in Color Images using a New Color Space Transformation. In: International Conference on Robotics and Biomimetics, pp. 737-741 (2004)

7. Periaswamy, S., Farid, H.: Elastic Registration in the Presence of Intensity Variations. IEEE Transactions on Medical Imaging 22(7), 865-874 (2003)

8. Shafer, S.A.: Using Color to Separate Reflection Components. Color Research and Application 10(4), 210-218 (1985)

9. Stoyanov, D., Yang, G.Z.: Removing Specular Reflection Components for Robotic Assisted Laparoscopic Surgery. In: International Conference on Image PRocessing, vol. 3, pp. 632-635 (2005)

10. Tan, R.T., Ikeuchi, K.: Separating Reflection Components of Textured Surfaces using a Single Image. IEEE Transactions on Pattern Analysis and Machine Intelligence 27(2), 178-193 (2005)

11. Tan, R.T., Nishino, K., Ikeuchi, K.: Illumination Chromaticity Estimation using Inverse-Intensity Chromaticity Space. In: Computer Vision and Pattern Recognition Conference, vol. 1, pp. 673-680 (2003) 